Uniqueness in inverse problems for an elasticity system with residual stress by a single measurement

This article has been downloaded from IOPscience. Please scroll down to see the full text article.

2003 Inverse Problems 19807

(http://iopscience.iop.org/0266-5611/19/4/301)

The Table of Contents and more related content is available

Download details:

IP Address: 140.112.113.225

The article was downloaded on 23/06/2009 at 12:53

Please note that terms and conditions apply. 


\title{
Uniqueness in inverse problems for an elasticity system with residual stress by a single measurement
}

\author{
Ching-Lung Lin ${ }^{1}$ and Jenn-Nan Wang ${ }^{2}$ \\ ${ }^{1}$ Department of Mathematics, National Cheng-Kung University, Tainan 701, Taiwan \\ 2 Department of Mathematics, National Taiwan University, Taipei 106, Taiwan \\ E-mail: jllin@math.ncku.edu.tw and jnwang@math.ntu.edu.tw
}

Received 5 November 2002, in final form 3 April 2003

Published 30 May 2003

Online at stacks.iop.org/IP/19/807

\begin{abstract}
In this paper we consider an elasticity system with residual stress. The constitutive equation of this elasticity system differs from that of the isotropic elasticity system by $R+(\nabla u) R$, where $R$ is the residual stress tensor. This system is not isotropic due to the existence of the residual stress $R$. Thus, it is not possible to reduce the principal part of the system to uncoupled wave operators as we have for the isotropic elasticity system. Here we investigate inverse problems of identifying the force term or the density by a single measurement of the lateral boundary. We establish uniqueness results by means of Carleman estimates when the residual stress is small.
\end{abstract}

\section{Introduction}

We consider a linear elasticity system with non-vanishing residual stress in this paper. Before describing the system, we want to define some notation which will be used throughout the paper. Let $Q$ be a cylindrical domain $(-T, T) \times \Omega$ in $\mathbb{R} \times \mathbb{R}^{3}$, where $\Omega$ is an open bounded domain in $\mathbb{R}^{3}$. Throughout we assume $0 \in \Omega$ and set $\omega=\sup _{x \in \Omega}|x|$. Use the notation $\Gamma=(-T, T) \times \partial \Omega$. Let $(t, x)=\left(t, x_{1}, x_{2}, x_{3}\right)$ be the coordinates in $\mathbb{R} \times \mathbb{R}^{3}$ and $\partial^{\alpha}=\partial_{t}^{\alpha_{0}} \partial_{1}^{\alpha_{1}} \partial_{2}^{\alpha_{2}} \partial_{3}^{\alpha_{3}}$ for any multi-index $\alpha=\left(\alpha_{0}, \alpha_{1}, \alpha_{2}, \alpha_{3}\right)$. Here $\partial_{j}=\frac{\partial}{\partial x_{j}}, j=1,2,3$. Also, we will set $\alpha^{\prime}=\left(0, \alpha_{1}, \alpha_{2}, \alpha_{3}\right)$. Unless otherwise indicated, we will use $\nabla, \nabla \cdot, \nabla \times$, and $\Delta$ to denote the gradient, divergence, curl, and Laplacian with respect to $x$-variables, respectively. All Latin indices are set to be from 1 to 3 whenever we do not specify their ranges. All Sobolev norms $\|\cdot\|_{s}$ not indicating the domain of integration are assumed to be integrating over $\mathbb{R}^{4}$. The letter $C$ stands for a generic constant whose value may vary from line to line.

The residual stress is modelled by a symmetric second-rank tensor $R(x)=\left(r_{j k}(x)\right) \in$ $C^{1}(Q)$ satisfying

$$
\nabla \cdot R=0 \quad \text { in } Q,
$$


where $\nabla \cdot R$ is a vector with components given by

$$
(\nabla \cdot R)_{j}=\sum_{k} \partial_{k} r_{j k}
$$

and

$$
R v=\sum_{k} r_{j k} v_{k}=0 \quad \text { on } \Gamma,
$$

where $v={ }^{t}\left(v_{1}, v_{2}, v_{3}\right)$ is the unit outer vector normal to $\partial \Omega$. Let $u(t, x)={ }^{t}\left(u_{1}, u_{2}, u_{3}\right)$ : $Q \rightarrow \mathbb{R}^{3}$ be the displacement vector; then the first Piola-Kirchhoff stress is

$$
\begin{gathered}
S(u)=R+(\nabla u) R+\tilde{\lambda}(\operatorname{tr} \epsilon) I+2 \tilde{\mu} \epsilon+\beta_{1}(\operatorname{tr} \epsilon)(\operatorname{tr} R) I+\beta_{2}(\operatorname{tr} R) \epsilon \\
+\beta_{3}((\operatorname{tr} \epsilon) R+\operatorname{tr}(\epsilon R) I)+\beta_{4}(\epsilon R+R \epsilon),
\end{gathered}
$$

where $\tilde{\lambda}(x), \tilde{\mu}(x)$ are the Lamé moduli, $\beta_{1}(x) \cdots \beta_{4}(x)$ are material parameters, and

$$
\epsilon=\frac{1}{2}\left(\nabla u+{ }^{t} \nabla u\right)
$$

is the strain tensor and $(\nabla u)_{j k}=\left(\partial_{k} u_{j}\right)$ (see [13]). In this paper we assume that $\beta_{3}=\beta_{4}=0$, i.e.

$$
S(u)=\lambda(\operatorname{tr} \epsilon) I+2 \mu \epsilon+R+(\nabla u) R
$$

where

$$
\lambda=\tilde{\lambda}+\beta_{1}(\operatorname{tr} R), \quad \mu=\tilde{\mu}+\frac{1}{2} \beta_{2}(\operatorname{tr} R) .
$$

We interrupt the exposition here to say a few words on the residual stress model. The constitutive equation (1.2) is close to the one considered by Robertson in [16] where he used the form

$$
S(u)=\tilde{\lambda}(\operatorname{tr} \epsilon) I+2 \tilde{\mu} \epsilon+R+(\nabla u) R
$$

to investigate the boundary determination of the residual stress by the Dirichlet-to-Neumann map. Hoger [4] also considered an elasticity system with residual stress where she used the constitutive equation

$$
S(u)=R+(\nabla u) R-\frac{1}{2}(\epsilon R+R \epsilon)+\tilde{\lambda}(\operatorname{tr} \epsilon) I+2 \tilde{\mu} \epsilon
$$

in her study. Based on Hartig's law, Man [13] argued that the constitutive equation of a realistic isotropic medium with residual stress should be given by (1.1) which describes a prestressed polycrystalline aggregate whose constituting crystallites are randomly oriented. Here we choose the constitutive equation (1.2) for studying related inverse problems for two reasons. On one hand, it is close to the realistic model as pointed out by Man. On the other hand, some basic properties for the elasticity system with the constitutive equation (1.2) have been established, especially Carleman estimates which lead to the uniqueness and stability of the Cauchy problem [9]. Those Carleman estimates play an important role in the study of some related inverse problems.

Henceforth, we denote

$$
\begin{aligned}
\mathcal{L} u & =\nabla \cdot S(u) \\
& \left.=(\lambda+\mu) \nabla(\nabla \cdot u)+\mu \Delta u-(\nabla \cdot u) \nabla \lambda-\left(\nabla u+{ }^{t}(\nabla u)\right) \nabla \mu-\nabla \cdot((\nabla u) R)\right) .
\end{aligned}
$$

Now let $y$ be a solution to the following initial boundary value problem

$$
\begin{array}{ll}
\rho \partial_{t}^{2} y-\mathcal{L} y=0 & \text { in } Q, \\
y(t, x)=\varphi(t, x) & \text { on } \Gamma, \\
y(0, x)=a(x) & \text { in } \Omega,
\end{array}
$$

where $\rho(x)>0$ is the density of the medium. In this paper, we are concerned with the inverse problem of determining the density $\rho(x)$ by measuring the traction of $y$ on $\Gamma$. 
Problem 1.1. Assume that coefficients $\tilde{\lambda}, \tilde{\mu}, \beta_{1}, \beta_{2}$, and $R$ are given. Can one uniquely determine $\rho(x)$ in $\Omega$ by measuring $\left.S(y) \nu\right|_{\Gamma}$ ? In other words, let $y$ and $\tilde{y}$ be solutions of (1.4) associated with densities $\rho$ and $\tilde{\rho}$. Does $\left.S(y) \nu\right|_{\Gamma}=\left.S(\tilde{y}) \nu\right|_{\Gamma}$ imply $\rho(x)=\tilde{\rho}(x)$ in $\Omega$ ?

Using the standard technique, see [11] for example, we can see that problem 1.1 is closely related to an inverse source problem. To be precise, let $u(t, x)$ be a solution solving

$$
\begin{array}{ll}
\rho \partial_{t}^{2} u-\mathcal{L} u=f(x) g(t, x) & \text { in } Q, \\
u(t, x)=0 & \text { on } \Gamma, \\
u(0, x)=0 & \text { in } \Omega,
\end{array}
$$

where $f(x)$ is a scalar function in $\Omega$ and $g(t, x)={ }^{t}\left(g_{1}(t, x), g_{2}(t, x), g_{3}(t, x)\right)$ is a vector function in $Q$. Then we consider the following inverse problem.

Problem 1.2. Let an appropriate $T>0$ be given. Does $\left.S(u) \nu\right|_{\Gamma}=0$ imply $f(x)=0$ in $\Omega$ ?

Notice that in (1.4) and (1.5), we do not provide $\partial_{t} y(0, x)$ and $\partial_{t} u(0, x)$ since they can also be determined in the related inverse problem (see $[7,11])$.

The proofs of uniqueness in problems 1.1 and 1.2 rely on Carleman estimates. The basic idea originated from Bukhgeim and Klibanov's paper [2]. After their paper, the uniqueness of similar inverse problems for hyperbolic equations based on Carleman estimates has been investigated—see, for example, [1, 6, 7, 10-12, 17].

The aforementioned results all dealt with a single hyperbolic equation. There were only a few attempts on systems of equations. For the isotropic elasticity system, an attempt has been made by Isakov [8] where he proved the Carleman estimate and established the uniqueness for the inverse source problem. It should be noted that in [8] Isakov transformed the principal part of the isotropic elasticity system to a composition of two scalar wave operators. For Maxwell's system, we mention Yamamoto's result in [18].

Our work is motivated by [5] in which Ikehata et al considered the isotropic elasticity system with variable coefficients. They used a different way to diagonalize the system. That is, they introduced an auxiliary function $\nabla \cdot u$ and transformed the principal part of the elasticity system to a diagonal system with wave operators as its diagonal components. The elasticity system that we consider here is not isotropic due to the existence of the residual stress. Therefore, the principal part cannot be reduced to uncoupled wave operators as we have for the isotropic elasticity system. Nevertheless, by introducing two auxiliary functions $\nabla \cdot u$ and $\nabla \times u$, we can transform the principal part to uncoupled wave operators plus second-order operators in $x$-variables acting only on $u$ with coefficients involving first derivatives of the residual stress. When the residual stress is assumed to be small, to take care of the additional second-order derivatives of $u$, we merely need a Carleman estimate for the Laplacian (see similar arguments in [9]).

One the other hand, for the determination of the density in problem 1.1, we only require one single measurement provided that the initial displacement satisfies an appropriate condition (see theorem 4.2). We would like to point out that for the inverse problem of identifying the density in [5], three measurements are needed in the three-dimensional case. We also want to compare our result with a result of Isakov [8] where he proved the uniqueness in determining the density by using four measurements in the three-dimensional case.

Finally, we would like to make some remarks on other related results in the parameter identification problem for the elasticity system. The first general result in this direction was proved by Nakamura and Uhlmann [14] in which they showed that two Lamé coefficients are uniquely determined by the static Dirichlet-to-Neumann map. In the dynamic setting, Rachele [15] proved that the finite-time Dirichlet-to-Neumann map uniquely determines 
the speeds of compressional and shear waves. Rachele's result implies that if one of the parameters, namely, the density function, with its Lamé coefficients, is known, then the other two parameters can be uniquely determined by the boundary map. It should be noted that results in $[14,15]$ require infinitely many boundary measurements. On the practical side, the unique determination of Lamé coefficients or Lamé coefficients plus density by means of finitely many boundary measurements has not been solved yet. Starting from this paper, we hope to pursue other interesting inverse problems of identifying parameters in the elasticity system by means of finitely many boundary measurements, even including the identification of residual stress.

The paper is organized as follows. In section 2, we transform the system in (1.5) to a larger system by introducing auxiliary functions $v=\nabla \cdot u$ and $w=\nabla \times u$. Since the system in (1.5) contains non-homogeneous terms, it is important to keep track of how non-homogeneous terms change in the process. Another key ingredient in solving problems 1.1 and 1.2 is the Carleman estimate. We will derive several useful Carleman estimates in section 3. In section 4, we will prove two theorems which will give definite answers to problems 1.1 and 1.2.

\section{Derivation of a new system}

Now, we suppose $u(t, x)$ is a solution of (1.5) and then rewrite $\nabla \cdot\{(\nabla u) R\}$ in (1.3) as

$$
\nabla \cdot\{(\nabla u) R\}=\sum_{j k} r_{j k} \partial_{j k}^{2} u+\nabla u(\nabla \cdot R)=\sum_{j k} r_{j k} \partial_{j k}^{2} u,
$$

since $\nabla \cdot R=0$. Dividing both sides of the first equation in (1.5) by $\rho$ yields

$$
\begin{gathered}
\partial_{t}^{2} u-\sum_{j k} r_{j k} \partial_{j k}^{2} u / \rho-(\lambda+\mu) / \rho \nabla(\nabla \cdot u)-\mu / \rho \Delta u-(\nabla \cdot u) \nabla \lambda / \rho \\
-\left(\nabla u+{ }^{t}(\nabla u)\right) \nabla \mu / \rho=(f / \rho) g .
\end{gathered}
$$

Let us introduce two auxiliary functions $v=\nabla \cdot u$ and $w=\nabla \times u$. Then we have from (2.1) that

$$
\begin{gathered}
\partial_{t}^{2} u-\sum_{j k} r_{j k} \partial_{j k}^{2} u / \rho-\mu / \rho \Delta u-(\lambda+\mu) / \rho \nabla v-\nabla \lambda / \rho v-\left(\nabla u+{ }^{t}(\nabla u)\right) \nabla \mu / \rho \\
=(f / \rho) g .
\end{gathered}
$$

Taking the divergence on (2.2) yields

$$
\begin{gathered}
\partial_{t}^{2} v-\sum_{j k} r_{j k} \partial_{j k}^{2} v / \rho-(\lambda+2 \mu) / \rho \Delta v-\sum_{j k} \nabla\left(r_{j k} / \rho\right) \cdot \partial_{j k}^{2} u-(\nabla(\mu / \rho)+\nabla \mu / \rho) \Delta u \\
-\{\nabla((\lambda+\mu) / \rho)+(\nabla \lambda+\nabla \mu) / \rho\} \cdot \nabla v-\{\nabla \cdot(\nabla \lambda / \rho)\} v \\
=(f / \rho)(\nabla \cdot g)+\nabla(f / \rho) \cdot g .
\end{gathered}
$$

By making the replacement

$$
\Delta u=\nabla(\nabla \cdot u)-\nabla \times(\nabla \times u)=\nabla v-\nabla \times w
$$

in (2.3), we get that

$$
\begin{aligned}
\partial_{t}^{2} v-\sum_{j k} r_{j k} \partial_{j k}^{2} v / \rho-(\lambda+2 \mu) / \rho \Delta v-\sum_{j k} \nabla\left(r_{j k} / \rho\right) \cdot \partial_{j k}^{2} u-(\nabla(\mu / \rho)+\nabla \mu / \rho) \\
\quad \times(\nabla v-\nabla \times w)-\{\nabla((\lambda+\mu) / \rho)+(\nabla \lambda+\nabla \mu) / \rho\} \cdot \nabla v-\nabla \cdot(\nabla \lambda / \rho) v \\
=(f / \rho)(\nabla \cdot g)+\nabla(f / \rho) \cdot g .
\end{aligned}
$$


Similarly, taking the curl of (2.2) and using the identities $\nabla \times(h u)=h \nabla \times u+\nabla h \times u$ and (2.4), we have that

$$
\begin{aligned}
\partial_{t}^{2} w-\sum_{j k} r_{j k} \partial_{j k}^{2} w / \rho-\mu / \rho \Delta w-\sum_{j k} \nabla\left(r_{j k} / \rho\right) \times \partial_{j k}^{2} u-\nabla(\mu / \rho) \times(\nabla v-\nabla \times w) \\
-\nabla((\lambda+\mu) / \rho) \times \nabla v-\nabla \times(\nabla \lambda / \rho) v-\nabla v \times(\nabla \lambda / \rho) \\
-\sum_{j}\left(\partial_{j} \mu / \rho \partial_{j} w+\nabla\left(\partial_{j} \mu / \rho\right) \times\left(\partial_{j} u+\nabla u_{j}\right)\right) \\
=(f / \rho) \nabla \times g+\nabla(f / \rho) \times g .
\end{aligned}
$$

Combining (2.2), (2.5), and (2.6) leads to the new system of equations

$$
\begin{aligned}
& \tilde{\square}_{r s} u+A_{1 ; 1}(u, v)=(f / \rho) g, \\
& \tilde{\square}_{r p} v-\sum_{j k} \nabla\left(r_{j k} / \rho\right) \cdot \partial_{j k}^{2} u+A_{2 ; 1}(u, v, w)=(f / \rho)(\nabla \cdot g)+\nabla(f / \rho) \cdot g, \\
& \tilde{\square}_{r s} w-\sum_{j k} \nabla\left(r_{j k} / \rho\right) \times \partial_{j k}^{2} u+A_{3 ; 1}(u, v, w)=(f / \rho) \nabla \times g+\nabla(f / \rho) \times g,
\end{aligned}
$$

where $\tilde{\square}_{r s}=\partial_{t}^{2}-\sum_{j k}\left(r_{j k}+\mu \delta_{j k}\right) / \rho \partial_{j k}^{2}, \tilde{\square}_{r p}=\partial_{t}^{2}-\sum_{j k}\left(r_{j k}+(\lambda+2 \mu) \delta_{j k}\right) / \rho \partial_{j k}^{2}$, and $A_{j ; 1}$ are first-order differential operators. In other words, we have proved the following proposition.

Proposition 2.1. Assume that $\lambda, \mu, \beta_{1}, \beta_{2}, R$ belong to $C^{2}(\bar{\Omega}), f$ and $\rho$ are in $C^{1}(\bar{\Omega})$, and $g(t, \cdot)$ belongs to $C^{1}(\bar{\Omega})$ for any $t \in(-T, T)$. Let $u \in H^{2}(Q)$ satisfy the first equation in (1.5) and $v=\nabla \cdot u$ and $w=\nabla \times u$. Then ${ }^{t}(u, v, w)$ satisfies the system of equations (2.7) and all first-order differential operators $A_{j ; 1}$ have bounded coefficients in $Q$.

In fact, to use the system (2.7) in our proofs, we need to express them in a more concrete form. Let us set $U={ }^{t}(u, v, w)$, which is a seven-dimensional vector function,

$$
\begin{aligned}
& \tilde{\square}_{2}\left(x, \partial_{x}, \partial_{t}\right)=\operatorname{diag}\left(\tilde{\square}_{r s}, \tilde{\square}_{r s}, \tilde{\square}_{r s}, \tilde{\square}_{r p}, \tilde{\square}_{r s}, \tilde{\square}_{r s}, \tilde{\square}_{r s}\right), \\
& R_{2}\left(x, \partial_{x}\right) u={ }^{t}\left(0,0,0, \sum_{j k} \nabla\left(r_{j k} / \rho\right) \cdot \partial_{j k}^{2} u, \sum_{j k} \nabla\left(r_{j k} / \rho\right) \times \partial_{j k}^{2} u\right), \\
& C_{1}\left(x, \partial_{x}\right) U={ }^{t}\left(A_{1 ; 1}\left(x, \partial_{x}\right)(u, v), A_{2 ; 1}\left(x, \partial_{x}\right)(u, v, w), A_{3 ; 1}\left(x, \partial_{x}\right)(u, v, w)\right), \\
& F(x)={ }^{t}\left(f / \rho, \partial_{1}(f / \rho), \partial_{2}(f / \rho), \partial_{3}(f / \rho), 0,0,0\right),
\end{aligned}
$$

and

$$
G(t, x)=\left(\begin{array}{ccccccc}
g_{1} & 0 & 0 & 0 & 0 & 0 & 1 \\
g_{2} & 0 & 0 & 0 & 0 & 1 & 0 \\
g_{3} & 0 & 0 & 0 & 0 & 0 & 0 \\
\partial_{1} g_{1}+\partial_{2} g_{2}+\partial_{3} g_{3} & g_{1} & g_{2} & g_{3} & 0 & 0 & 0 \\
\partial_{2} g_{3}-\partial_{3} g_{2} & 0 & g_{3} & -g_{2} & 0 & 0 & 0 \\
\partial_{3} g_{1}-\partial_{1} g_{3} & -g_{3} & 0 & g_{1} & 0 & 0 & 0 \\
\partial_{1} g_{2}-\partial_{2} g_{1} & g_{2} & -g_{1} & 0 & 1 & 0 & 0
\end{array}\right)
$$

then the system of equations (2.7) can be written as

$$
\tilde{\square}_{2} U-R_{2} u+C_{1} U=G F .
$$

It should be noted that the first four columns of $G$ are determined by the right-hand side of (2.7). Here we extend $G$ into a $7 \times 7$ square matrix by adding the last three columns. Of course, they can be arbitrary since the last three components of $F$ are zero. The reason for choosing these three columns in the above form is that we can compute the determinant of $G$ explicitly. Indeed, by direct computations, we get that

$$
\operatorname{det} G(t, x)=g_{3}(t, x)^{2}|g(t, x)|^{2}=g_{3}(t, x)^{2}\left(g_{1}(t, x)^{2}+g_{2}(t, x)^{2}+g_{3}(t, x)^{2}\right) .
$$


Therefore, if we assume

$$
g_{3}(0, x)^{2}>0 \quad x \in \bar{\Omega}
$$

and $g \in C^{1}(\bar{Q})$, then there exists a positive constant $\zeta$ such that the inverse matrix $G^{-1}(t, x)$ of $G(t, x)$ exists and $\operatorname{det} G(t, x)>0$ for $x \in \bar{\Omega}$ and $|t| \leqslant \zeta$.

Remark 2.1. By playing with the last three columns of $G(t, x)$, the condition (2.9) can be replaced by $g_{1}(0, x)^{2}>0$ or $g_{2}(0, x)^{2}>0$. We thank the referee for pointing out this feature to us.

\section{Carleman estimates}

Our idea for solving problems 1.1 and 1.2 is based on Carleman estimates. In this section, we would like to derive appropriate Carleman estimates for the wave and Laplacian operators. To begin, let $P\left(y, D_{y}\right)$ be an $m$ th-order differential operator with real coefficients having principal symbol $p(y, \xi)$.

Definition 3.1. A $C^{2}$-function $\phi(y)$ is called strongly pseudo-convex with respect to $P$ on $\Lambda$ at $y_{0}$ if it satisfies

$$
\begin{aligned}
& \left\{p_{-\tau}, p_{\tau}\right\} / \tau i \geqslant C\left(|\xi|^{2}+\tau^{2}\right) \quad \text { for some constant } C>0 \\
& \text { on }\left\{p_{\tau}\left(y_{0}, \xi\right)=0,\left(y_{0}, \xi\right) \in \Lambda, \tau \geqslant 0,(\xi, \tau) \neq 0\right\},
\end{aligned}
$$

where $\{\cdot, \cdot\}$ is the Poisson bracket, $p_{\tau}\left(y_{0}, \xi\right)=p\left(y_{0}, \xi+i \tau \nabla_{y} \phi\left(y_{0}\right)\right)$, and $\Lambda$ is a subset of $T_{y_{0}}^{*} \mathbb{R}^{n}$.

Here we will consider the operator $P_{c}\left(t, x, \partial_{t}, \partial_{x}\right)=\partial_{t}^{2}-\sum_{j k}\left(r_{j k} / \rho+c \delta_{j k}\right) \partial_{j k}^{2}$ for $c=\mu / \rho$ or $c=(\lambda+2 \mu) / \rho$. We assume that the residual stress satisfies

$$
\sum_{j k}\left\|\frac{r_{j k}}{\rho}\right\|_{C^{1}}<\varepsilon
$$

for some constant $0<\varepsilon<1$. By assuming the ellipticity condition on the Lamé parameters $\tilde{\lambda}$ and $\tilde{\mu}$, i.e.

$$
\tilde{\mu}>0 \quad \text { and } \quad \tilde{\lambda}+2 \tilde{\mu}>0 \quad \text { for all }(t, x) \in \bar{Q},
$$

we can get that

$$
\mu>0 \quad \text { and } \quad \lambda+2 \mu>0 \quad \text { for all }(t, x) \in \bar{Q},
$$

provided that $\varepsilon$ is sufficiently small. Introducing the weight function

$$
\phi(t, x)=\exp ((\sigma / 2) \psi(t, x))
$$

where

$$
\psi(t, x)=x_{1}^{2}+x_{2}^{2}+x_{3}^{2}-\theta t^{2} .
$$

Denote $Q_{\delta}=Q \cap\{\psi>\delta\}$, where $\delta>0$. In what follows we will prove that $\phi(t, x)$ is strongly pseudo-convex with respect to $P_{c}$ on $\mathbb{R}^{4}$ in $Q_{\delta}$ for large $\sigma$ and small $\varepsilon$ when $c$ satisfies some restrictions.

Proposition 3.1. Assume that $\left(r_{j k}\right)$ satisfies the estimate (3.2). Let $c(x) \in C^{1}$ satisfy

$$
\theta c+\theta \sqrt{c}|t \nabla c|+\frac{1}{2} c \nabla c \cdot x<c^{2}+\frac{1}{2} \theta t\left(\partial_{t} c\right)+\frac{1}{2} c b\left(\partial_{3} c\right)
$$

and

$$
0<\theta c^{-1} \leqslant 1-\left(\min _{\bar{Q}} c\right)^{-1} \varepsilon
$$

for $(t, x) \in \bar{Q}$. Then $\phi(t, x)$ is strongly pseudo-convex with respect to $P_{c}$ on $\mathbb{R}^{4}$ in $Q_{\delta}$ provided that $\sigma$ is sufficiently large and $\varepsilon$ is small enough. 
Proof. A thorough proof of the proposition was given in [9] (see the proof of proposition 3.1 in [9]). So we omit it here.

Having derived the pseudo-convexity condition for the weight function $\phi(t, x)$, we can discuss the corresponding Carleman estimate for $\tilde{\square}_{r s / r p}$. The following form is adopted from [9] and it can be proved by the same arguments as in [3] (see the proof of theorem 1.3 in [3]).

Theorem 3.1. Let $\mu \in C^{1}(\bar{Q})\left(\right.$ or $\left.\lambda, \mu \in C^{1}(\bar{Q})\right)$ and $\rho, R=\left(r_{j k}\right) \in C^{1}(\bar{Q})$. Assume that $\mu / \rho($ or $(\lambda+2 \mu) / \rho)$ satisfies (3.4) and (3.5). Then we can find a small constant $\varepsilon_{0}>0$ such that if $R$ satisfies (3.2) with $\varepsilon<\varepsilon_{0}$, then there exist constants $C\left(\varepsilon_{0}, \delta\right)$ and $C_{0}\left(\varepsilon_{0}, \delta, \sigma\right)$ such that

$$
\left\|\eta^{\frac{3}{2}-|\alpha|} \mathrm{e}^{\tau \phi} \partial^{\alpha} u\right\|_{0} \leqslant C\left\|\mathrm{e}^{\tau \phi} \tilde{\square}_{r s / r p} u\right\|_{0}
$$

for all $u \in C_{0}^{\infty}\left(Q_{\delta}\right)$ provided $|\alpha| \leqslant 1, \sigma \geqslant C$, and $\tau \geqslant C_{0}$. Here $\eta=\tau \sigma \phi$ and the small constant $\varepsilon_{0}$ depends on $C^{1}$-norms of coefficients $\lambda, \mu$, and $\rho$.

In the following we will need a Carleman estimate for $\Delta$ in terms of the weight function $\phi$. This result has been proved in [3] (see theorem 1.1 in [3]).

Theorem 3.2. There exist constants $C(\delta)$ and $C_{0}(\delta, \sigma)$ such that

$$
\sqrt{\sigma}\left\|\eta^{\frac{3}{2}-\left|\alpha^{\prime}\right|} \mathrm{e}^{\tau \phi} \partial^{\alpha^{\prime}} u\right\|_{0} \leqslant C\left\|\mathrm{e}^{\tau \phi} \Delta u\right\|_{0}
$$

for any $u \in C_{0}^{\infty}\left(Q_{\delta}\right)$ provided that $\left|\alpha^{\prime}\right| \leqslant 2, \sigma \geqslant C$, and $\tau \geqslant C_{0}$.

Remark 3.1. It is readily seen that the Carleman estimates (3.6) and (3.7) are valid for $u \in H_{0}^{2}\left(Q_{\delta}\right)$.

\section{Main results}

We will solve problems 1.1 and 1.2 in this section. We first study problem 1.2 and prove theorem 4.1 below. Having solved problem 1.2, the solution to problem 1.1 follows immediately by the standard argument.

Theorem 4.1. Suppose that all coefficients $\tilde{\lambda}, \tilde{\mu}, \beta_{1}, \beta_{2}$ are in $C^{2}(\bar{Q})$ and $R, \rho, g$ are in $C^{3}(\bar{Q})$. Let $\mu / \rho$ and $(\lambda+2 \mu) / \rho$ satisfy conditions (3.4) and (3.5). Let $g$ satisfy (2.9), i.e.

$$
g_{3}(0, x)^{2}>0 \quad x \in \bar{\Omega} .
$$

Assume that $\Omega \subset B(0, \sqrt{\theta} T)$ and $S(u)(t, x) v=0$ on $\Gamma$. Then there exists an $\varepsilon_{0}>0$ such that if $R$ satisfies (3.2) with $\varepsilon<\varepsilon_{0}$, then for the solution $u \in C^{3}(\bar{Q})$ to (1.5), one has $f(x)=0$ in $\Omega$ and $u(x, t)=0$ in $Q_{0}$.

Proof. As described in section 2, we transform the first equation in (1.5) to the system (2.8). By virtue of (2.9), we know that $G^{-1}(t, x)$ exists for $x \in \bar{\Omega},|t| \leqslant \zeta$, with sufficiently small $\zeta$. Therefore, multiplying both sides of $(2.8)$ by $G^{-1}$ yields

$G^{-1}(t, x) \tilde{\square}_{2}\left(x, \partial_{x}, \partial_{t}\right) U-G^{-1}(t, x) R_{2}\left(x, \partial_{x}\right) u+G^{-1}(t, x) C_{1}\left(x, \partial_{x}\right) U=F(x)$

or $x \in \bar{\Omega},|t| \leqslant \zeta$. Note that the right-hand side of (4.1) is independent of $t$. So differentiating both sides of (4.1) with respect to $t$ gives

$$
\begin{aligned}
\left(\partial_{t} G^{-1}(t, x)\right) \tilde{\square}_{2}\left(x, \partial_{x}, \partial_{t}\right) U+G^{-1}(t, x) \tilde{\square}_{2}\left(x, \partial_{x}, \partial_{t}\right) \partial_{t} U-\left(\partial_{t} G^{-1}(t, x)\right) R_{2}\left(x, \partial_{x}\right) u & \\
& -G^{-1}(t, x) R_{2}\left(x, \partial_{x}\right) \partial_{t} u+\left(\partial_{t} G^{-1}(t, x)\right) C_{1}\left(x, \partial_{x}\right) U \\
& +G^{-1}(x, t) C_{1}\left(x, \partial_{x}\right) \partial_{t} U=0 .
\end{aligned}
$$


Now, multiplying both sides of (4.2) by $G(t, x)$ and denoting $A(t, x)=G(t, x) \partial_{t} G^{-1}(t, x)$, we get that

$$
\tilde{\square}_{2} \partial_{t} U+A \tilde{\square}_{2} U-R_{2} \partial_{t} u-A R_{2} u+C_{1} \partial_{t} U+A C_{1} U=0 .
$$

It follows from (4.3) that

$$
\tilde{\square}_{2}\left(\partial_{t} U+A U\right)=R_{2} \partial_{t} u+A R_{2} u-C_{1}\left(\partial_{t} U+A U\right)-\left[A, \tilde{\square}_{2}\right] U-\left[A, C_{1}\right] U
$$

or

$$
\tilde{\square}_{2}(N U)=R_{2} \partial_{t} u+A R_{2} u-C_{1}(N U)-\left[A, \tilde{\square}_{2}\right] U-\left[A, C_{1}\right] U,
$$

where $N U=\partial_{t} U+A U$ and $[\cdot, \cdot]$ is the commutator notation.

By direct computations and lemma A.2 in the appendix, we have that

$$
\begin{gathered}
\left\|\mathrm{e}^{\tau \phi}\left[A, \tilde{\square}_{2}\right] U+\mathrm{e}^{\tau \phi}\left[A, C_{1}\right] U\right\|_{0}\left(\tilde{Q}_{\delta}\right) \leqslant C \sum_{|\alpha| \leqslant 1}\left\|\mathrm{e}^{\tau \phi} \partial^{\alpha} U\right\|_{0}\left(\tilde{Q}_{\delta}\right) \\
\leqslant C \sum_{\left|\alpha^{\prime}\right| \leqslant 1}\left\|\mathrm{e}^{\tau \phi} \partial^{\alpha^{\prime}}(N U)\right\|_{0}\left(\tilde{Q}_{\delta}\right),
\end{gathered}
$$

where $\tilde{Q}_{\delta}=Q_{\omega^{2}-\theta \zeta^{2}+\delta}$ with $\delta>0$ sufficiently small. Recall that $\omega=\sup _{x \in \Omega}|x|$ and $\alpha^{\prime}=\left(0, \alpha_{1}, \alpha_{2}, \alpha_{3}\right)$. It should be noted that the commutator $\left[A, \tilde{\square}_{2}\right]$ is of order one.

Now we are going to apply the Carleman estimates (3.6) and (3.7) to $N U$ and $\partial_{t}^{n} u, n=0,1$, respectively. However, we cannot directly work on $N U$ and $\partial_{t}^{n} u$ since they may not satisfy the support condition. To overcome this difficulty, we want to introduce a suitable cut-off function. Let $\chi \in C^{\infty}(Q)$ be a cut-off function with $0 \leqslant \chi \leqslant 1$ and

$$
\chi= \begin{cases}1, & \text { in } Q_{\omega^{2}-\theta \zeta^{2}+\delta}, \\ 0, & \text { in } Q \backslash Q_{\left(\omega^{2}-\theta \zeta^{2}\right)} ;\end{cases}
$$

then we need to show that $\chi N U \in H_{0}^{2}\left(\tilde{Q}_{0}\right)$ and $\chi \partial_{t}^{n} u \in H_{0}^{2}\left(\tilde{Q}_{0}\right), n=0,1$. To verify these properties, it suffices to prove $\left.\partial^{\alpha} N U\right|_{\Gamma}=\left.\partial^{\alpha} \partial_{t}^{n} u\right|_{\Gamma}=0$ for $|\alpha| \leqslant 1$ and $n=0,1$. To do so, we will make use of lemma A.1 in the appendix. Thus, what we should do is to check whether $\rho \partial_{t}^{2} u-\mathcal{L} u=0$ on $\Gamma$. The condition clearly holds if we show that $f(x)=0$ on $\partial \Omega$. In fact, by virtue of the zero Dirichlet data and $u \in C^{3}(\bar{Q})$, we have that

$$
-\mathcal{L} u=f(x) g(t, x) \quad \text { on } \Gamma .
$$

Consequently, setting $t=0$ in (4.7), the zero initial condition in (1.5) and the assumption on $g(t, x)$ (see (2.9)) imply that $f(x)=0$ on $\partial \Omega$.

Since both $\mu / \rho$ and $(\lambda+2 \mu) / \rho$ satisfy (3.4) and (3.5), we can apply the estimate (3.6) to $\chi N U$ as long as the residual stress is small in the sense of (3.2). Here and below, in using (3.6) and (3.7) it suffices to take $\sigma$ to be sufficiently large and then fixed. In view of (4.4) and (4.5), we hence obtain that for large $\tau$,

$$
\begin{aligned}
& \sum_{|\alpha| \leqslant 1} \tau^{\frac{3}{2}-|\alpha|}\left\|\mathrm{e}^{\tau \phi} \partial^{\alpha}(N U)\right\|_{0}\left(\tilde{Q}_{\delta}\right) \leqslant \sum_{|\alpha| \leqslant 1} \tau^{\frac{3}{2}-|\alpha|}\left\|\mathrm{e}^{\tau \phi} \partial^{\alpha}(\chi N U)\right\|_{0}\left(\tilde{Q}_{0}\right) \\
& \leqslant\left\|\mathrm{e}^{\tau \phi} \tilde{\square}_{2}(\chi N U)\right\|_{0}\left(\tilde{Q}_{0}\right) \\
& \leqslant C \varepsilon \sum_{\left|\alpha^{\prime}\right|=2}\left\|\mathrm{e}^{\tau \phi} \partial^{\alpha^{\prime}}\left(\partial_{t} u\right)\right\|_{0}\left(\tilde{Q}_{0}\right)+C \varepsilon \sum_{\left|\alpha^{\prime}\right|=2}\left\|\mathrm{e}^{\tau \phi} \partial^{\alpha^{\prime}} u\right\|_{0}\left(\tilde{Q}_{0}\right) \\
&+C \sum_{|\alpha| \leqslant 1}\left\|\mathrm{e}^{\tau \phi} \partial^{\alpha}(N U)\right\|_{0}\left(\tilde{Q}_{0}\right)+C \sum_{|\alpha| \leqslant 1}\left\|\mathrm{e}^{\tau \phi} \partial^{\alpha} U\right\|_{0}\left(\tilde{Q}_{0}\right) \\
& \leqslant C \varepsilon \sum_{\left|\alpha^{\prime}\right|=2}\left\|\mathrm{e}^{\tau \phi} \partial^{\alpha^{\prime}}\left(\partial_{t} u\right)\right\|_{0}\left(\tilde{Q}_{\delta}\right)+C \varepsilon \sum_{\left|\alpha^{\prime}\right|=2}\left\|\mathrm{e}^{\tau \phi} \partial^{\alpha^{\prime}} u\right\|_{0}\left(\tilde{Q}_{\delta}\right)
\end{aligned}
$$




$$
\begin{aligned}
& \quad+C \sum_{|\alpha| \leqslant 1}\left\|\mathrm{e}^{\tau \phi} \partial^{\alpha}(N U)\right\|_{0}\left(\tilde{Q}_{\delta}\right)+C \varepsilon \sum_{\left|\alpha^{\prime}\right|=2}\left(\left\|\mathrm{e}^{\tau \phi} \partial^{\alpha^{\prime}}\left(\partial_{t} u\right)\right\|_{0}\left(\tilde{Q}_{\delta}^{c}\right)+\left\|\mathrm{e}^{\tau \phi} \partial^{\alpha^{\prime}} u\right\|_{0}\left(\tilde{Q}_{\delta}^{c}\right)\right) \\
& +C \sum_{|\alpha| \leqslant 1}\left(\left\|\mathrm{e}^{\tau \phi} \partial^{\alpha}(N U)\right\|_{0}\left(\tilde{Q}_{\delta}^{c}\right)+\left\|\mathrm{e}^{\tau \phi} \partial^{\alpha} U\right\|_{0}\left(\tilde{Q}_{\delta}^{c}\right)\right) \\
& \leqslant C \varepsilon \sum_{\left|\alpha^{\prime}\right|=2}\left\|\mathrm{e}^{\tau \phi} \partial^{\alpha^{\prime}}\left(\partial_{t} u\right)\right\|_{0}\left(\tilde{Q}_{\delta}\right)+C \varepsilon \sum_{\left|\alpha^{\prime}\right|=2}\left\|\mathrm{e}^{\tau \phi} \partial^{\alpha^{\prime}} u\right\|_{0}\left(\tilde{Q}_{\delta}\right) \\
& \quad+C \varepsilon \sum_{\left|\alpha^{\prime}\right|=2}\left(\left\|\mathrm{e}^{\tau \phi} \partial^{\alpha^{\prime}}\left(\partial_{t} u\right)\right\|_{0}\left(\tilde{Q}_{\delta}^{c}\right)+\left\|\mathrm{e}^{\tau \phi} \partial^{\alpha^{\prime}} u\right\|_{0}\left(\tilde{Q}_{\delta}^{c}\right)\right) \\
& \quad+C \sum_{|\alpha| \leqslant 1}\left(\left\|\mathrm{e}^{\tau \phi} \partial^{\alpha}(N U)\right\|_{0}\left(\tilde{Q}_{\delta}^{c}\right)+\left\|\mathrm{e}^{\tau \phi} \partial^{\alpha} U\right\|_{0}\left(\tilde{Q}_{\delta}^{c}\right)\right),
\end{aligned}
$$

where $\tilde{Q}_{0}=Q_{\omega^{2}-\theta \zeta^{2}}$ and $\tilde{Q}_{\delta}^{c}=Q_{\omega^{2}-\theta \zeta^{2}} \backslash Q_{\omega^{2}-\theta \zeta^{2}+\delta}$. Here the integral of the zeroth and first derivative terms of $N U$ over $\tilde{Q}_{\delta}$ has been absorbed into the left-hand side of the inequality by taking $\tau$ large enough.

Now we want to take care of the second derivatives of $\partial_{t} u$ and $u$ on the right-hand side of (4.8). We first consider the term with $\partial_{t} u$. By virtue of the Carleman estimate (3.7) and the obvious vector identity $\Delta\left(\partial_{t} u\right)=\nabla\left(\partial_{t} v\right)-\nabla \times\left(\partial_{t} w\right)$, we have that

$$
\begin{aligned}
& \sum_{\left|\alpha^{\prime}\right|=2}\left\|\mathrm{e}^{\tau \phi} \partial^{\alpha^{\prime}}\left(\partial_{t} u\right)\right\|_{0}\left(\tilde{Q}_{\delta}\right) \leqslant \sum_{\left|\alpha^{\prime}\right|=2}\left\|\mathrm{e}^{\tau \phi} \partial^{\alpha^{\prime}}\left(\chi \partial_{t} u\right)\right\|_{0}\left(\tilde{Q}_{0}\right) \leqslant C \tau^{1 / 2}\left\|\mathrm{e}^{\tau \phi} \Delta\left(\chi \partial_{t} u\right)\right\|_{0}\left(\tilde{Q}_{0}\right) \\
& \leqslant C \tau^{1 / 2}\left\|\mathrm{e}^{\tau \phi} \Delta\left(\partial_{t} u\right)\right\|_{0}\left(\tilde{Q}_{\delta}\right)+C \tau^{1 / 2}\left\|\mathrm{e}^{\tau \phi} \Delta\left(\chi \partial_{t} u\right)\right\|_{0}\left(\tilde{Q}_{\delta}^{c}\right) \\
& \leqslant C \tau^{1 / 2}\left\|\mathrm{e}^{\tau \phi} \nabla\left(\partial_{t} v\right)\right\|_{0}\left(\tilde{Q}_{\delta}\right)+C \tau^{1 / 2}\left\|\mathrm{e}^{\tau \phi} \nabla \times\left(\partial_{t} w\right)\right\|_{0}\left(\tilde{Q}_{\delta}\right) \\
&+C \tau^{1 / 2}\left\|\mathrm{e}^{\tau \phi} \Delta\left(\chi \partial_{t} u\right)\right\|_{0}\left(\tilde{Q}_{\delta}^{c}\right) \\
& \leqslant C \tau^{1 / 2} \sum_{\left|\alpha^{\prime}\right| \leqslant 1}\left\|\mathrm{e}^{\tau \phi} \partial^{\alpha^{\prime}}\left(\partial_{t} U\right)\right\|_{0}\left(\tilde{Q}_{\delta}\right)+C \tau^{1 / 2}\left\|\mathrm{e}^{\tau \phi} \Delta\left(\chi \partial_{t} u\right)\right\|_{0}\left(\tilde{Q}_{\delta}^{c}\right) \\
& \leqslant C \tau^{1 / 2} \sum_{\left|\alpha^{\prime}\right| \leqslant 1}\left\|\mathrm{e}^{\tau \phi} \partial^{\alpha^{\prime}}(N U)\right\|_{0}\left(\tilde{Q}_{\delta}\right)+C \tau^{1 / 2} \sum_{\left|\alpha^{\prime}\right| \leqslant 1}\left\|\mathrm{e}^{\tau \phi} \partial^{\alpha^{\prime}} U\right\|_{0}\left(\tilde{Q}_{\delta}\right) \\
&+C \tau^{1 / 2}\left\|\mathrm{e}^{\tau \phi} \Delta\left(\chi \partial_{t} u\right)\right\|_{0}\left(\tilde{Q}_{\delta}^{c}\right) \\
& \leqslant C \tau^{1 / 2} \sum_{\left|\alpha^{\prime}\right| \leqslant 1}\left\|\mathrm{e}^{\tau \phi} \partial^{\alpha^{\prime}}(N U)\right\|_{0}\left(\tilde{Q}_{\delta}\right)+C \tau^{1 / 2}\left\|\mathrm{e}^{\tau \phi} \Delta\left(\chi \partial_{t} u\right)\right\|_{0}\left(\tilde{Q}_{\delta}^{c}\right) .
\end{aligned}
$$

Note that for getting the last two inequalities of (4.9) we have used the definition of $N U$ and the estimate (4.5). Going over the same arguments, we can obtain that

$$
\sum_{\left|\alpha^{\prime}\right|=2}\left\|\mathrm{e}^{\tau \phi} \partial^{\alpha^{\prime}} u\right\|_{0}\left(\tilde{Q}_{\delta}\right) \leqslant C \tau^{1 / 2} \sum_{\left|\alpha^{\prime}\right| \leqslant 1}\left\|\mathrm{e}^{\tau \phi} \partial^{\alpha^{\prime}}(N U)\right\|_{0}\left(\tilde{Q}_{\delta}\right)+C \tau^{1 / 2}\left\|\mathrm{e}^{\tau \phi} \Delta(\chi u)\right\|_{0}\left(\tilde{Q}_{\delta}^{c}\right) .
$$

Combining (4.8), (4.9), (4.10) and taking $\varepsilon$ sufficiently small, we obtain that

$$
\begin{aligned}
\sum_{|\alpha| \leqslant 1} \tau^{\frac{3}{2}-|\alpha|}\left\|\mathrm{e}^{\tau \phi} \partial^{\alpha}(N U)\right\|_{0}\left(\tilde{Q}_{\delta}\right) \leqslant C \varepsilon \tau^{1 / 2}\left\|\mathrm{e}^{\tau \phi} \Delta(\chi u)\right\|_{0}\left(\tilde{Q}_{\delta}^{c}\right) \\
+C \varepsilon \tau^{1 / 2}\left\|\mathrm{e}^{\tau \phi} \Delta\left(\chi \partial_{t} u\right)\right\|_{0}\left(\tilde{Q}_{\delta}^{c}\right)+C \varepsilon \sum_{\left|\alpha^{\prime}\right|=2}\left(\left\|\mathrm{e}^{\tau \phi} \partial^{\alpha^{\prime}}\left(\partial_{t} u\right)\right\|_{0}\right. \\
\left.+\left\|\mathrm{e}^{\tau \phi} \partial^{\alpha^{\prime}} u\right\|_{0}\right)\left(\tilde{Q}_{\delta}^{c}\right)+C \sum_{|\alpha| \leqslant 1}\left(\left\|\mathrm{e}^{\tau \phi} \partial^{\alpha}(N U)\right\|_{0}+\left\|\mathrm{e}^{\tau \phi} \partial^{\alpha} U\right\|_{0}\right)\left(\tilde{Q}_{\delta}^{c}\right) .
\end{aligned}
$$

Now we observe that $\phi>\mathrm{e}^{\sigma\left(\omega^{2}-\theta \zeta^{2}+\delta\right) / 2}$ on $\tilde{Q}_{\delta}$ and $\phi \leqslant \mathrm{e}^{\sigma\left(\omega^{2}-\theta \zeta^{2}+\delta\right) / 2}$ on $\tilde{Q}_{\delta}^{c}$. Thus, replacing $\phi$ on the left- and right-hand sides of (4.11) by its minimum and maximum values over the corresponding domains of integration and dividing both sides by $\exp \left(\tau \mathrm{e}^{\sigma\left(\omega^{2}-\theta \zeta^{2}+\delta\right) / 2}\right)$ leads to 


$$
\begin{aligned}
\tau^{\frac{3}{2}}\|N U\|_{0}\left(\tilde{Q}_{\delta}\right) & \leqslant C \varepsilon \tau^{1 / 2}\|\Delta(\chi u)\|_{0}\left(\tilde{Q}_{\delta}^{c}\right)+C \varepsilon \tau^{1 / 2}\left\|\Delta\left(\chi \partial_{t} u\right)\right\|_{0}\left(\tilde{Q}_{\delta}^{c}\right) \\
& +C \varepsilon \sum_{\left|\alpha^{\prime}\right|=2}\left(\left\|\partial^{\alpha^{\prime}}\left(\partial_{t} u\right)\right\|_{0}+\left\|\partial^{\alpha^{\prime}} u\right\|_{0}\right)\left(\tilde{Q}_{\delta}^{c}\right) \\
& +C \sum_{|\alpha| \leqslant 1}\left(\left\|\partial^{\alpha}(N U)\right\|_{0}+\left\|\partial^{\alpha} U\right\|_{0}\right)\left(\tilde{Q}_{\delta}^{c}\right) .
\end{aligned}
$$

Dividing both sides of (4.12) by $\tau^{3 / 2}$ and letting $\tau \rightarrow \infty$, we immediately have that $N U=0$ in $Q_{\omega^{2}-\theta \zeta^{2}+\delta}$. From $N U=0$ we get $\partial_{t}\left(G^{-1} U\right)=0$ in $Q_{\omega^{2}-\theta \zeta^{2}+\delta}$. Taking into account the zero initial condition in (1.5), we conclude that $u=0$ in $Q_{\omega^{2}-\theta \zeta^{2}+\delta}$. Since $\delta>0$ is arbitrarily small, we obtain

$$
\begin{aligned}
& u(t, x)=0, \quad(t, x) \in Q_{\omega^{2}-\theta \zeta^{2}}, \\
& f(x)=0, \quad \sqrt{\omega^{2}-\theta \zeta^{2}} \leqslant|x| \leqslant \omega .
\end{aligned}
$$

Next we will expand the domain where the uniqueness holds. To do this, we want to extend $G$ in $t$ to $\tilde{G}$ so that $\tilde{G}^{-1}$ exists in this larger domain and equation (2.8) holds with $G$ being replaced by $\tilde{G}$. We define a function $\kappa(t) \in C^{\infty}(\mathbb{R})$ satisfying $0 \leqslant \kappa(t) \leqslant 1$ and

$$
\kappa(t)= \begin{cases}0, & \text { in }|t| \leqslant \zeta \\ 1, & \text { in }|t|>\gamma \zeta\end{cases}
$$

where $\gamma>1$ and is very close to 1 . Use the notation $\tilde{G}(t, x)=(1-\kappa(t)) G(t, x)+\kappa(t) G(0, x)$, i.e.

$$
\tilde{G}(t, x)= \begin{cases}G(t, x), & \text { if }|t| \leqslant \zeta \\ G(0, x), & \text { if }|t|>\gamma \zeta\end{cases}
$$

Now for $|t| \leqslant \gamma \zeta$ we can see that

$$
\|\tilde{G}(t, x)-G(t, x)\|=\|\kappa(t)(G(0, x)-G(t, x))\| \leqslant\left\|\partial_{t} G\right\|_{L^{\infty}(\bar{Q})}(\gamma \zeta) .
$$

Therefore, we can choose $\zeta>0$ such that (2.9) holds,

$$
\operatorname{det} \tilde{G}(t, x)>0 \quad x \in \bar{\Omega},|t| \leqslant \gamma \zeta
$$

and

$$
\zeta=\frac{T}{\sqrt{m}} \quad \text { for some } m \in \mathbb{N}
$$

On the other hand, one can easily check that if $(t, x) \in Q_{\omega^{2}-2 \theta \zeta^{2}} \cap\left\{(t, x):|x| \leqslant \sqrt{\omega^{2}-\theta \zeta^{2}}\right\}$, then $|t| \leqslant \zeta$. This implies that

$$
\tilde{G} F=G F \quad \text { in } Q_{\omega^{2}-2 \theta \zeta^{2}} \cap\left\{(t, x):|x| \leqslant \sqrt{\omega^{2}-\theta \zeta^{2}}\right\} .
$$

Furthermore, we know that $f(x)=0$ for $\sqrt{\omega^{2}-\theta \zeta^{2}} \leqslant|x| \leqslant \omega$, which leads to

$$
\tilde{G} F=G F=0 \quad \text { in } Q_{\omega^{2}-2 \theta \zeta^{2}} \cap\left\{(t, x): \sqrt{\omega^{2}-\theta \zeta^{2}} \leqslant|x| \leqslant \omega\right\} .
$$

Therefore, we can see that

$$
\tilde{G} F=G F \quad \text { in } Q_{\omega^{2}-2 \theta \zeta^{2}} \cap\{(t, x):|x| \leqslant \omega\}=Q_{\omega^{2}-2 \theta \zeta^{2}} .
$$

Recall that $\omega=\sup _{x \in \Omega}|x|$. In other words, we obtain from (2.8) that

$$
\begin{aligned}
& \tilde{\square}_{2} U-R_{2} u+C_{1} U=\tilde{G} F, \quad(t, x) \in Q_{\omega^{2}-2 \theta \zeta^{2}}, \\
& u(t, x)=0, \quad S(u) v(t, x)=0 \quad \text { on } \partial Q_{\omega^{2}-2 \theta \zeta^{2}} \cap \Gamma, \\
& u(0, x)=0 \quad \text { in } \Omega
\end{aligned}
$$


and $\tilde{G}^{-1}(t, x)$ exists in $\bar{Q}_{\omega^{2}-2 \theta \zeta^{2}}$. Hence, we can repeat the above argument and get that

$$
\begin{array}{ll}
u(t, x)=0, & (t, x) \in Q_{\omega^{2}-2 \theta \zeta^{2}}, \\
f(x)=0, & \sqrt{\omega^{2}-2 \theta \zeta^{2}} \leqslant|x| \leqslant \omega .
\end{array}
$$

By repeating the above argument $m$ times, we have that

$$
\begin{array}{ll}
u(t, x)=0, & (x, t) \in Q_{\omega^{2}-m \theta \zeta^{2}}, \\
f(x)=0, & \sqrt{\omega^{2}-m \theta \zeta^{2}} \leqslant|x| \leqslant \omega .
\end{array}
$$

It follows from (4.14) that $\omega^{2}-m \theta \zeta^{2}=\omega^{2}-\theta T^{2}<0$ since $\bar{\Omega} \subset B(0, \sqrt{\theta} T)$. Consequently, we have that $f(x)=0$ for $x \in \Omega$ and $u(t, x)=0$ for $(t, x) \in Q_{0}$.

Theorem 4.2. Suppose that all coefficients $\tilde{\lambda}, \tilde{\mu}, \beta_{1}, \beta_{2}$ are in $C^{2}(\bar{Q})$ and $R, \rho, \tilde{\rho}$ are in $C^{3}(\bar{Q})$. Let $y$ and $\tilde{y}$ be solutions of (1.4) corresponding to density functions $\rho$ and $\tilde{\rho}$, respectively. Assume that $\mu / \rho$ and $(\lambda+2 \mu) / \rho$ satisfy conditions (3.4), (3.5), and $\Omega \subset B(0, \sqrt{\theta} T)$. Let the initial condition $a(x)$ satisfy

$$
\mathcal{L}_{3}(a)^{2}>0 \quad \text { in } \bar{\Omega},
$$

where $\mathcal{L}_{3}(a)$ denotes the third component of the vector $\mathcal{L}(a)$. Then there exists an $\varepsilon_{0}>0$ such that if $R$ satisfies (3.2) with $\varepsilon<\varepsilon_{0}$ and $\left.S(y) \nu\right|_{\Gamma}=\left.S(\tilde{y}) \nu\right|_{\Gamma}$, then one has $\rho(x)=\tilde{\rho}(x)$ in $\Omega$ and $y(t, x)=\tilde{y}(t, x)$ in $Q_{0}$.

Proof. Let $u=y-\tilde{y}, f(x)=\frac{\tilde{\rho}(x)-\rho(x)}{\tilde{\rho}(x)}$ and $g(t, x)=\tilde{\rho}(x) \partial_{t}^{2} \tilde{y}(t, x)$; then we can get that

$$
\begin{array}{lcc}
\rho \partial_{t}^{2} u-\mathcal{L}(u)=f(x) g(t, x) \quad \text { in } Q, & \\
u(t, x)=0, & S(u)(t, x) v(x)=0 & \text { on } \Gamma, \\
u(0, x)=0 & \text { in } \Omega .
\end{array}
$$

Also, one can check that

$$
g(0, x)=\tilde{\rho}(x) \partial_{t}^{2} \tilde{y}(0, x)=\mathcal{L}(u(0, x))=\mathcal{L}(a) .
$$

Thus, equation (4.15) implies (2.9). Now theorem 4.2 follows directly from theorem 4.1 .

\section{Acknowledgment}

This work was partially supported by the Taiwan National Science Council NSC 91-2115-M002-019.

\section{Appendix}

Lemma A.1. Let $u \in C^{3}(\bar{Q})$ satisfy

$$
\rho \partial_{t}^{2} u-\mathcal{L} u=u(t, x)=S(u) v(t, x)=0 \quad \text { on } \Gamma
$$

and $\|R\|_{C^{0}}(\bar{Q})=r \ll 1$; then

$$
\frac{\partial u}{\partial x_{k}}=\frac{\partial^{2} u}{\partial x_{j} \partial x_{i}}=0 \quad \text { on } \Gamma .
$$

Therefore,

$$
U=(u, v, w)=(u, \nabla \cdot u, \nabla \times u)=N U=\frac{\partial(N U)}{\partial v}=0 \quad \text { on } \Gamma,
$$

where $N U=\partial_{t} U-A(x, t) U$. 
Proof. Recall that $S(u)=\lambda(\operatorname{tr} \epsilon) I+2 \mu \epsilon+R+(\nabla u) R$ and $R v=0$ on $\Gamma$. Since $u(t, x)=0$ on $\Gamma$, it is clear that

$$
\nabla u_{i}=\frac{\partial u_{i}}{\partial v} v \quad \text { on } \Gamma .
$$

Componentwise, we have that

$$
\frac{\partial u_{i}}{\partial x_{j}}=\frac{\partial u_{i}}{\partial v} v_{j} \quad \text { on } \Gamma .
$$

By direct computations, we get for $(t, x) \in \Gamma$ that

$$
\begin{aligned}
0 & =(\nabla u) v \cdot S(u) v \\
& =\lambda(\operatorname{tr} \epsilon)^{2}+\mu(\operatorname{tr} \epsilon)^{2}+\mu\left(\Sigma_{i}\left\|\nabla u_{i}\right\|^{2}\right) \\
& =(\lambda+2 \mu)(\operatorname{tr} \epsilon)^{2}+\mu\left(\Sigma_{i}\left\|\nabla u_{i}\right\|^{2}-(\operatorname{tr} \epsilon)^{2}\right) .
\end{aligned}
$$

The ellipticity condition (3.3) and $\Sigma_{i=1}^{3}\left\|\nabla u_{i}\right\|^{2}-(\operatorname{tr} \epsilon)^{2} \geqslant 0$ imply

$$
\operatorname{tr} \epsilon=0 \quad \text { and } \quad \nabla u_{i}=0 \quad \forall i .
$$

Thus, $\frac{\partial u_{i}}{\partial x_{k}}=0$ on $\Gamma$ for all $i, k$. As above, we find that

$$
\nabla\left(\frac{\partial u_{i}}{\partial x_{j}}\right)=\frac{\partial^{2} u_{i}}{\partial \nu \partial x_{j}} v \quad \text { on } \Gamma .
$$

Componentwise, we have

$$
\frac{\partial^{2} u_{i}}{\partial x_{j} \partial x_{k}}=v_{j} v_{k} \frac{\partial^{2} u_{i}}{\partial v^{2}} \quad \text { on } \Gamma \text {. }
$$

For convenience, we denote $b_{i}=\frac{\partial^{2} u_{i}}{\partial v^{2}}$ and $b=\left(b_{1}, b_{2}, b_{3}\right)$. Since $u(t, x)=\frac{\partial u_{i}}{\partial x_{k}}=0$ on $\Gamma$ and $R$ satisfies (3.2), we get for $(t, x) \in \Gamma$ that

$$
\begin{aligned}
0 & =b \cdot \mathcal{L} u \\
& \geqslant(\lambda+\mu)(b \cdot v)^{2}+\mu\|b\|^{2}-r\|b\|^{2} \\
& =(\lambda+2 \mu-r)(b \cdot v)^{2}+(\mu-r)\left(\|b\|^{2}-(b \cdot v)^{2}\right) .
\end{aligned}
$$

Now the ellipticity condition (3.3) and $r \ll 1$ imply that

$$
b \cdot v=0 \quad \text { and } \quad b_{i}=0 \quad \forall i .
$$

In view of (A.1), we have that $\frac{\partial^{2} u_{i}}{\partial x_{j} \partial x_{i}}=0$ on $\Gamma$ for all $i, j, k$ and, therefore, $U=N U=$ $\frac{\partial(N U)}{\partial v}=0$ on $\Gamma$. Note that $\partial_{t} U=0$ on $\Gamma$ is obvious.

Lemma A.2. Let $A(t, x)$ be a $7 \times 7$ matrix with $A(t, x) \in C^{2}([-\zeta, \zeta] \times \Omega)$ and $V(t, x) \in$ $C^{1}([-\zeta, \zeta] \times \Omega)$ satisfying $V(0, x)=0$; then we have the following estimate:

$$
\sum_{|\alpha| \leqslant 1}\left\|\mathrm{e}^{\tau \phi} \partial^{\alpha}(V)\right\|_{0}\left(\tilde{Q}_{\delta}\right) \leqslant C \sum_{\left|\alpha^{\prime}\right| \leqslant 1}\left\|\mathrm{e}^{\tau \phi} \partial^{\alpha^{\prime}}(N V)\right\|_{0}\left(\tilde{Q}_{\delta}\right)
$$

where $\tilde{Q}_{\delta}$ and the operator $N$ are defined as in the proof of theorem 4.1.

Proof. This lemma was essentially proved in [5]. We reproduce the proof here for the sake of completeness. Recall that

$$
\partial_{t} V+A V=N V
$$


Let the matrix function $K(s, t, x) \in C^{2}\left(\left\{(s, t, x):(t, x) \in \tilde{Q}_{\delta}, 0<s<t\right\}\right)$ be the fundamental solution of the differential equation (A.2) in $t$ such that

$$
V(t, x)=\int_{0}^{t} K(s, t, x)(N V)(s, x) \mathrm{d} s, \quad(t, x) \in \tilde{Q}_{\delta} .
$$

From (A.3) we get that

$$
\begin{gathered}
\partial_{x_{i}} V(t, x)=\int_{0}^{t} \partial_{x_{i}} K(s, t, x)(N V)(s, x) \mathrm{d} s+\int_{0}^{t} K(s, t, x) \partial_{x_{i}}(N V)(s, x) \mathrm{d} s, \\
(t, x) \in \tilde{Q}_{\delta}
\end{gathered}
$$

and

$$
\begin{aligned}
\partial_{t} V(t, x) & =-A(t, x) V(t, x)+(N V)(t, x) \\
& =(N V)(t, x)-\int_{0}^{t} A(t, x) K(s, t, x)(N V)(s, x) \mathrm{d} s, \quad(t, x) \in \tilde{Q}_{\delta} .
\end{aligned}
$$

Therefore, we have that, for all $(t, x) \in \tilde{Q}_{\delta}$,

$$
\sum_{|\alpha| \leqslant 1}\left|\partial^{\alpha} V(t, x)\right| \leqslant C|(N V)(t, x)|+C \sum_{\left|\alpha^{\prime}\right| \leqslant 1} \int_{0}^{t}\left|\partial^{\alpha^{\prime}}(N V)(s, x)\right| \mathrm{d} s .
$$

Thus,

$$
\sum_{|\alpha| \leqslant 1}\left\|\mathrm{e}^{\tau \phi} \partial^{\alpha}(V)\right\|_{0}\left(\tilde{Q}_{\delta}\right) \leqslant C\left\|\mathrm{e}^{\tau \phi}(N V)\right\|_{0}\left(\tilde{Q}_{\delta}\right)+C \sum_{\left|\alpha^{\prime}\right| \leqslant 1}\left\|\mathrm{e}^{\tau \phi} \int_{0}^{t}\left|\partial^{\alpha^{\prime}}(N V)(s, x)\right| \mathrm{d} s\right\|_{0}\left(\tilde{Q}_{\delta}\right) \text {. }
$$

Now, we recall the following estimate proved in [5] (see [5, appendix]):

$$
\sum_{\left|\alpha^{\prime}\right| \leqslant 1}\left\|\mathrm{e}^{\tau \phi} \int_{0}^{t}\left|\partial^{\alpha^{\prime}}(N V)(s, x)\right| \mathrm{d} s\right\|_{0}\left(\tilde{Q}_{\delta}\right) \leqslant C \sum_{\left|\alpha^{\prime}\right| \leqslant 1}\left\|\mathrm{e}^{\tau \phi} \partial^{\alpha^{\prime}}(N V)\right\|_{0}\left(\tilde{Q}_{\delta}\right) .
$$

The proof is now completed if we replace the last term of (A.4) by (A.5).

\section{References}

[1] Bukhgeim A L 1988 Introduction to the Theory of Inverse Problems (Novosibirsk: Nauka)

[2] Bukhgeim A L and Klibanov M V 1988 Global Uniqueness of a Class of Multidimensional Inverse Problems (Novosibirsk: Nauka) (Engl. transl. 1981 Sov. Math.-Dokl. 24 244-7)

[3] Eller M and Isakov V 2000 Carleman estimates with two large parameters and applications Proc. Conf. AMS Summer Research (Boulder, CO, July 1999), Contemp. Math. 268 117-37

[4] Hoger A 1986 On the determination of residual stress in an elastic body J. Elast. 16 303-24

[5] Ikehata M, Nakamura G and Yamamoto M 1998 Uniqueness in inverse problems for the isotropic Lame system J. Math. Sci. Univ. Tokyo 5 627-92

[6] Isakov V 1989 Uniqueness of the continuation across a time-like hyperplane and related inverse problems for hyperbolic equations Commun. Partial Diff. Eqns 14 465-78

[7] Isakov V 1990 Inverse Source Problems (Mathematical Surveys and Monographs No 34) (Providence, RI: American Mathematical Society)

[8] Isakov V 1986 A nonhyperbolic Cauchy problem for $\square_{b} \square_{c}$ and its applications to elasticity theory Commun. Pure Appl. Math. 39 747-67

[9] Isakov V, Nakamura G and Wang J N Uniqueness and stability in the Cauchy problem for the elasticity system with residual stress Contemp. Math. at press

[10] Khă̌darov A 1987 Carleman estimates and inverse problems for second order hyperbolic equations Math. USSR Sbornik 58 267-77

[11] Klibanov M 1992 Inverse problems and Carleman estimates Inverse Problems 8575-96 
[12] Kubo M 1995 Identification of the potential term of the wave equation Proc. Japan Acad. 71 174-6

[13] Man C S 1998 Hartig's law and linear elasticity with initial stress Inverse Problems 14 313-9

[14] Nakamura G and Uhlmann G 1994 Global uniqueness for an inverse boundary problem arising in elasticity Inv. Math. 118 457-74

[15] Rachele L 2000 An inverse problem in elastodynamics: uniqueness of the wave speeds in the interior J. Diff. Eqns 162 300-25

[16] Robertson R 1997 Boundary identifiability of residual stress via the Dirichlet to Neumann map Inverse Problems $131107-19$

[17] Yamamoto M 1999 Uniqueness and stability in multidimensional hyperbolic inverse problems J. Math. Pure Appl. 78 65-98

[18] Yamamoto M 1998 On an inverse problem of determining source terms in Maxwell's equations with a single measurement Inverse Problems, Tomography, and Image Processing (Newark, DE, 1997) (New York: Plenum) pp 241-56 